\title{
ENFERMEDADES INFECTO-CONTAGIOSAS Y TUBERCULOSIS INFANTIL
}

Pot los Dres.

\author{
JORGE PEÑA CERECEDA.
}

Tissólogo del Hospital Atriarán. Médico-Jefe del Sanatorio "Los Guindos" JULIO MENEGHELLO.

Médico residente del Sanatorio de "Los Guindos"

y GASTON GONZALEZ D.

Ayudante del Servicio.

(Conclusión)

\section{Escarlatina y tuberculosis}

Desde mucho tiempo atrás se ha considerado que la es. carlatina no ejerce ninguna acción sobre la tuberculosis, ya sea ésta activa o inactiva. Rilliet y Barthez llegan aún más iejos: afirman que la escarlatina y la tuberculosis son enfermedades antagónicas y que la primeta ejerce una acción beneficiosa sobre la segunda, cuando ambas se asocian. Dufourt. y Viallier ( 5 y 6 ) dicen que la escarlatina no ejerce ninguna acción sobre los procesos tuberculosos con lesiones congestivas o sobre la epituberculosis, pero que hay que ser más teservado en lo que se refiere a las tuberculosis caseosas of $i$ brocaseosas, pues estas formas suelen agravarse con ocasión de una escarlatina. Nalbant (9), en 13 casos de escarlatina $Y$ tuberculosis estudiados, encontró agravación de esta última sólo en un caso. Choffe (7), en 178 casos de escarlatina del sanatorio de enfermedades infecciosas de Hendaya, la mayoría de los cuales tenían lesiones francas tuberculosas, no enconuró más que dos niños que agravaron su tuberculosis. Piccaluga (20), dice que la escarlatina hace negativa casi siempre la reacción a la tuberculina, pero que no ejerce influencia especial sobre la tuberculosis. Pilcher (8), dice que en la escarlatína la reacción a la tuberculina es más débil mientras mayor gea la intensidad del "rash" escarlatiniforme; y explica este 


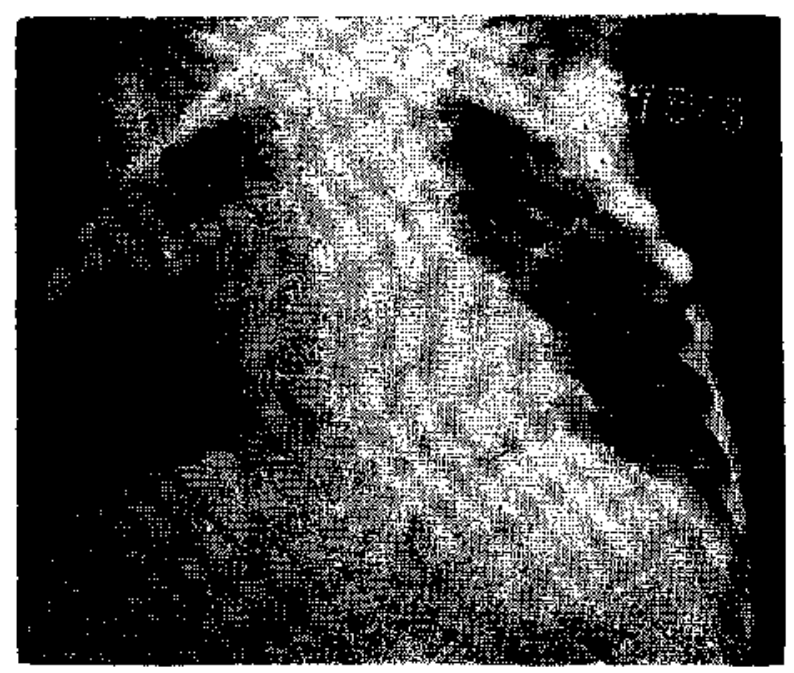

CASO N.: 13,-S. O. FIG. 7.-Radiogtafía tomada ef $8-\mathrm{X}-38$, antes de la coqueluche: adenopatía
paratraqueal superior bilateral.

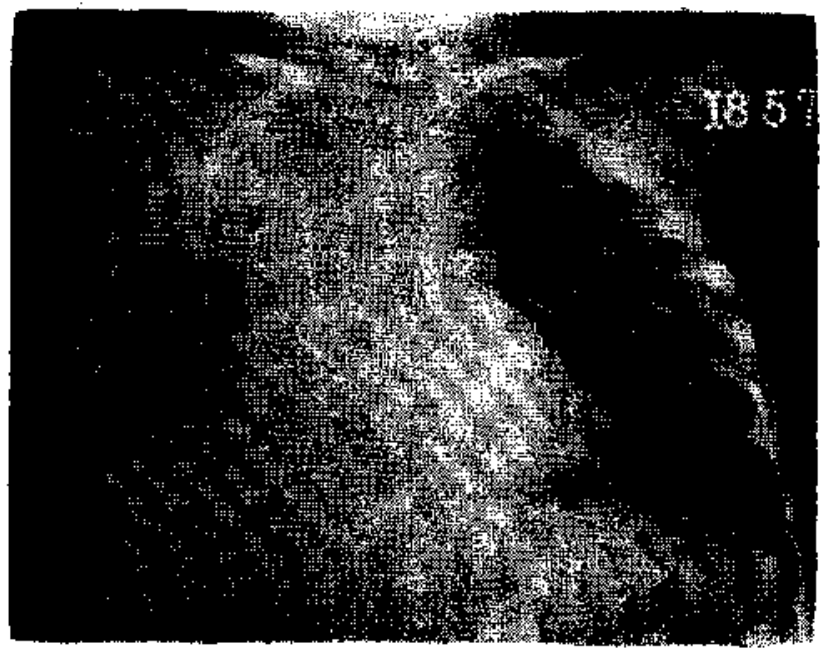

FIG. 8.-Radiografia tomada en enero de 1939, 1 mes después de la eoquelathe: 42. observa una cxtension de las lesiones. 
fenómeno por trastornos locales de la piel y tejidos vecinos, especialmente de la circulación.

En cambio. Millian y Vassiliu $(21$ y 22$)$, dicen que muy a menudo la escarlatina agrava la tuberculosis y viceversa. En un estudio que los autores hicieron en un hospital de niños de Bucarest, observaron que en la mayoría de los casos de escarlatina grave, que llevaban a la muerte, se encontraban en la autopsia lesiones tuberculosas diseminadas (en e1 $50 \%$, según los autores). Presentan 9 casos de escarlatina maligna, en los que, en la autopsia se encontraron lesiones agudas tuberculosas. Estos niños eran aparentemente sanos antes de la aparición de la escarlatina. Millian explica esta agravación de la siguiente manera: "se ha visto que la escarlatina se acompaña habitualmente de fenómenos de insuficiencia suprarrenal; se sabe también que la tuberculosis provoca constantemente una insuficiencia suprarrenal relativa (astenia, hipotensión): además, tanto la escarlatina como la tuberculosis producen una alteración hepática. El sindroma maligno resultaría pues, de la acción convergente de las dos infecciones sobre los mismos órganos (higado y suprarrenales) que, como se sabe, tienen un gran rol antitóxico". (22).

Roger. Widal y Teissier (23), dicen también que la escarlatina tiene a menudo una acción desfavorable sobre el curso de la tuberculosis y que hace negativa la cutirreacción a la tuberculina. Brandenberg dice lo mismo. Igual cosa, en Alemania, Schöf, Fanconi y Teissierj.

En general, podemos decir que la relación entre escarlatina y tuberculosis no ha sido tan bien estudiada como la existente entre esta última enfermedad con el sarampión o la coqueluche.

Nosotros hemos estudiado la relación entre escarlatina y tuberculosis en 23 casos. En el cuadro que va a continuación se exponen los resultados obtenidos por nosotros.

\begin{tabular}{|c|c|c|c|}
\hline $\begin{array}{l}\text { N. }{ }^{\circ} \text { de } \\
\text { casos }\end{array}$ & & $\begin{array}{l}\text { Influidos } \\
\text { desfavorablemente }\end{array}$ & $\begin{array}{l}\text { No } \\
\text { influídos }\end{array}$ \\
\hline 12 & Con lesiones TBC anteriores .... & $2(10 \%)$ & $10(43 \%)$ \\
\hline i1 & $\begin{array}{l}\text { Aparición de lesiones TBC conse- } \\
\text { cutivamente a excarlatioa } \ldots . . .\end{array}$ & $11(47 \%)$ & \\
\hline 23 & $\begin{array}{lllllll}\text { TOTAL } & \ldots & \ldots & \ldots & \ldots & \ldots . & \ldots\end{array}$ & $12(57 \%)$ & $10(43 \%)$ \\
\hline
\end{tabular}

Como vemos, los resultados obtenidos son muy semejantes a los que hemos visto a propósito del sarampión y la coqueluche: en más de la mitad de los casos la escarlatina 


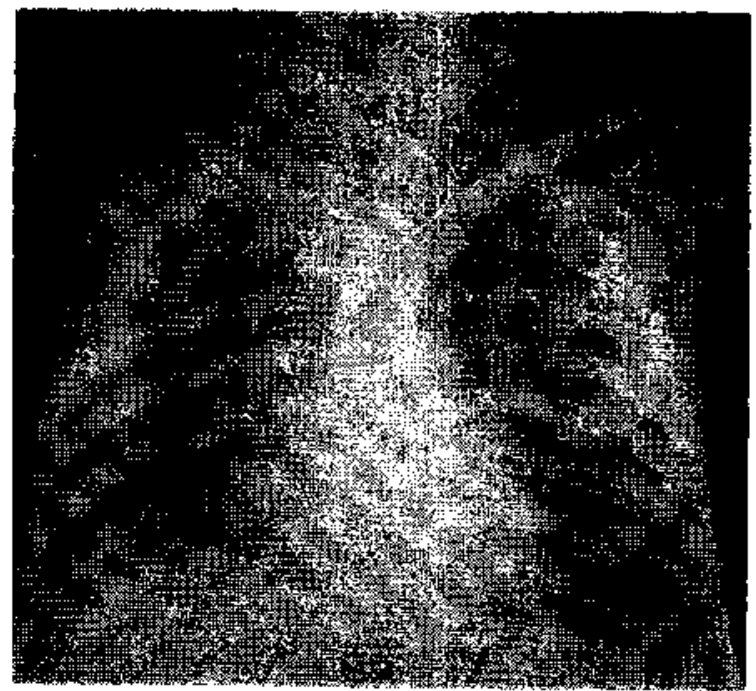

CASO N. $14-\mathrm{H} . \mathrm{P}$.

FIG. 9,--Radiografia tomada en mayo de 1938, antes de la escariatina: adenopatía paratraquesl derecha.

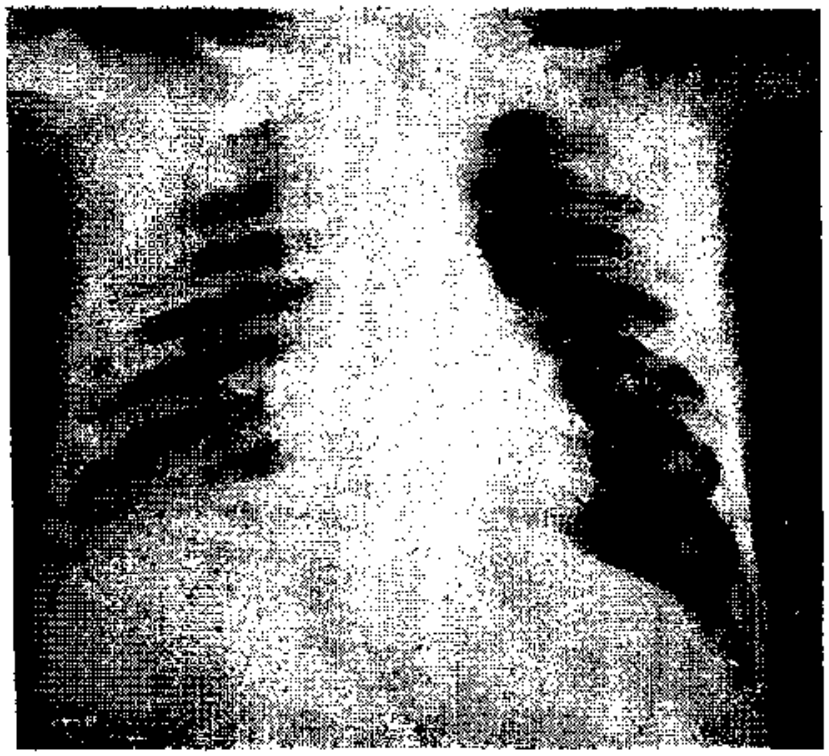

FIG. 10.--Radiografía tomada el 15-XI-3B, 3 meses despuéz de la escarlatina: infiltrado de la mitad superior derecha. 
influye desfavorablemente a la tuberculosis, ya sea ésta evolutiva o latente. En dos casos, el proceso evolucionó hacia la muerte. Gran parte de las agravaciones son sólo temporales. Los casos agravados corresponden en su mayoría a formas activas de tuberculosis, y los to agravados, a formas inactivas.

Desgraciadamente. no tenemos datos suficientes para pronunciarnos sobre el comportamiento de la reacción a la tuberculina durante la escarlatina. Sólo podemos decir que en tres oportunidades encontramos un Mantoux positivo en ple. na escarlatina y cinco veces. negativo.

A continuación exponemos algunos casos de agravación de la tuberculosis por la escarlatina.

CASO N.? 14,-H. P. Obs. 160.1938. Edad: 4 años.

Anterdentes.-No se tiene ningún dato sobre ás: es huétfano y viene de provincias.

Ingresa al Servicio de Cirugia del Hospilu Arriaran a 16 de cnero de 1938. por una ósteoarticis ulcerada de la rodilla dececha. por lo que hubo necesidad de amputar cl muslo de sc lado. Se tastada al Servicio de Tubareulogis por una odenoparia paratraqueal con mediana actividad $y$ estasionaria.

Contrae una escarlationa en agosto de 1938 que. a pesar de su evolución benigna, activa el proceso y produce un aumento munifieste de li combra mediastinal superior del lado detecho. La sedimantación sube de 30-70 mns. a 100 120 mms. Apartén dizas térmicas que persisten durante dos meses. Curva de peso estacionaria. I. a sadiografia 17298. tomada cl 15 de septirmbre de 19? 8 . semuestra, ademá; de la adenopatis, ила infiltración d: la mitad superior dekc tha.

Sólo tres meses después comienzan a declinar las temperacutas. y a los cinco mezes se logra norma'izar Is sedimentación. Persiste a la pantalla la imagen de adenopatia.

E: do advertir que este nifio ha estado sometido a un régimen fanatoria! en "Los Guindos", darante casi todo el curso de su enfermedad.

Comentario.--Se trata de un niño de 4 años con un proceso ósteoarticular fistulizado de la rodilla derecha, por el cual se le amputa el muslo. y que presenta además una adenopatía paratraqueal poco activa, que después de una escarlatina benigna se agrava y el proceso se extiende al parenquima pulmonar. Esta agravación cede después de 6 meses de tratamıento climático.

CASO N.9 15.-L. V. Obs. 1385. 1934. Edad: 12 años.

Antecedentes.-La madre mutió de taberculosiz pulmonari una tis. que ha convivido con la niña durante varios meses, falleció de tuberealosis puImonar en el Hospital San José. bace algunos meses. 
Ingreia al Servicio de Tuberculosis del Hospital Arriarán el 19 de mapo de 1934. Hace más o menos an año que la chica decae visiblemente: enflaquece, disminuye su apetito, está pálida y adinámica. I.a tos, seca al principio, en el úlima tiempo se acompaña de desgarro nucopara'ento y que ea algunas oca. siones es bemoptoiso.

Ultimamente ha tenido + hemoprisis. El examen revela ura tubercalosis exudativa de ambos vértices y mal estado general. Sedimentación: $54.77 \mathrm{~mm}$. Baciloscopia positiva. En noviemb:e del mismo año contra tna escarlatina que agrava considerablemente el estado general y anmenta las lesiones pulmonares, principalmente la izquierda, Se da el alta a pedido de la familia en condiciones muy malas.

CASO N.9 16.-M. A. Obs. 03619. Edad: 8 años.

Antecedentes.-Padre rouerto de toberculosis pulmonar. Alimentada en matas condiciones.

Enfo'medad actoal. - Se inscribe en el Policlínico del Hospital "Manuel Arriarán". en mayo de 1937, por un Pirquet positivo y una conjuntivitis flictenular. En diciembre del mismo año contrae una escatlatina complicada de bronconemmonia. Un mes despú́s se compraeba un infiltrado tuberculoso derecho. Esta lesión siguió una evolución favorable y en abril de 1938 era casi imperceptible.

Comentario.-Por la evolución tan rápida de esta lesión pulmonar (4 meses) nos inclinamos a pensar en una atelectasia. Se trataría en este caso de una acción de la escarlatina sobre la adenopatía tráqueo-brónquica e indirectamente sobre el pulmón, produciendo la atelectasia.

CASO N.9 17.-E. S. Obs. 482. 1939. Edad: 3 años.

Antecedentes.-Madre tuberculosa.

Enfermedad actual,--Ingresa al Secvicio de Infectiosos del Hospital "Maruel Arriarán" en febreto de 1939, convaleciente de escarlatina y, con el diagnóstico de bronconeumonia. Lo5 exámenes clínicos $y$ radiológicos indican una condensación de todo el pu'món izguierdo, reblandecida. Sadimentación: 65-105 ma. Alta a pedido de la familia en estado grave.

Comentario.- En esta enfermita se hace el diagnóstico de neumonia caseosa, la que sólo se revela a taíz de una escarlatina. Los datos anteriores no permiten hacer pensar en la existencia de una lesión tuberculosa, por lo menos de la extensión de la que se comprueba en esta niña después de la enfermedad intercurrente.

Antes de seguir el estudio de otras enfermedades infecto contagiosas en su relación con la tuberculosis, queremos llamar la atención sobre el siguiente hecho: tanto el sarampión 
como la coqueluche y la escarlatina, en nuestra estadística dan un crecido porcentaje, alrededor del $40 \%$, de procesos tuberculosos consecutivos a la enfermedad infecciosa; y en cambio. este potcentaje apenas alcanza al $15 \%$ de los casos de tuberculosis preexistentes y agravadas por la enfermedad intercurrente. Este hecho, en apariencia contradictorio, no es tal si tomamos en cuenta que la gran mayoría de los casos en que el proceso tuberculoso ha sido secundario a la enfermedad infecriosa, ha sido reclutada en el Servicio de Infecciosos, y se trata de niños que no han sido estudiados anteriormente desde el punto de vista pulmonar: estamos seguros, que la gran mayoria de ellos, tenían una tuberculosis estacionaria antes de la aparición de la enfermedad intercurrente.

Hecha esta aclaración. seguimos estudiando la influencia de otras enfermedades infecto-contagiosas sobre la tuberculosis.

\section{Varicela y tuberculosis}

En general, la varicela nunca ha sido considerada como enfermedad anergizante $y$ no se ha señalado una influencia especial de ella sobre la tuberculosis activa o latente.

Nalbant, en su trabajo tantas veces citado (9), estudia 39 casos de varicela, de los cuales 21 tenían una tuberculosis activa y 17 una forma latente; sólo en tres casos, o sea el $7,5 \%$, encontró que la tuberculosis se agravaba por la va. ticela. En ningún caso notó un cambio en la tuberculinoreacción durante el período álgido de la varicela.

Georg Abraham (24), dice que la varicela no ejerce ninguna acción sobre la tuberculosis. En cambio. W. Schwenk (25), afirma que la varicela ejerce una acción desfavorable sobre la tuberculosis, y que cuando aparece en un niño tuberculoso, éste fácilmente hace una meningitis tuberculosa. Este peligro se acentúa en los niños mayores de seis años, y que tienen una forma grave de tuberculosis.

En la Argentina, el Dr. Generoso Schiavone (26), dice que la relación entre la tuberculosis y varicela ha sido poco estudiada; agrega que Rieux y Combe le niegan a la varicela todo poder anergizante y que en cambio Mohr y Staehlin di. cen que en el $3 \%$ de los casos, la tuberculosis aparece consecutivamente a una varicela. El autor muestra un caso de meningitis tuberculosa, que aparece después de una varicela en un chico aparentemente sano antes de esta última enfermedad. Termina citando la opinión de Schönfeld. quien, en 8 niños 
tuberculosos que hicieron una varicela, encontró en dos de ellos una abolición de la tuberculino-reacción, en dos una dis. minución fuerte, en dos una disminución débil y en los otros tres, la reacción persistió igual que antes de la varicela.

Dufourt y Viallier (5), dicen que la varicela no ejerce ninguna influencia sobre la tuberculosis. Choffe (7), en 238 casos del sanatorio de Hendaya, no encontró en ninguno de ellos agravación de la tuberculosis por la varicela.

Nosotros hemos estudiado 13 casos de varicela; los resultados obtenidos van en el cuadro siguiente:

\begin{tabular}{|c|c|c|c|}
\hline $\begin{array}{l}\text { NA. }{ }^{\circ} \text { de } \\
\operatorname{cas} \operatorname{sos}\end{array}$ & & $\begin{array}{c}\text { Influidos } \\
\text { desfavorablemente }\end{array}$ & $\begin{array}{l}\text { No } \\
\text { inflnídos }\end{array}$ \\
\hline 11 & Con lesiones TBC antcriotes & $(8 \%)$ & $10(76 \%)$ \\
\hline 2 & $\begin{array}{l}\text { Aparición de lesiones TBC conse- } \\
\text { cotivamente a varicela } \ldots . . .\end{array}$ & $2(16 \%)$ & \\
\hline 13 & TOTAL . & $3\left(24 w_{n}^{\prime}\right)$ & $10(76 \%)$ \\
\hline
\end{tabular}

Podemos ver que en la gran mayoria de los casos la va. ricela no ejerció ninguna influencia sobre la tuberculosis, y que la agravó sólo en el $24 \%$ de los casos. Las agravaciones fueron todas temporales y no hubo ningún caso de falle. cimiento por ellas. En 4 casos la reacción a la tuberculina persistió positiva durante la varicela, y en un caso se hizo negativa. habiendo sido antes positiva. Como vemos, nuestra estadística está más o menos de acuerdo con lo que dicen los autores que hemos citado.

\section{Difteria y tubercalosis}

Es muy poca la literatura que hemos encontrado respecto a este tema. Dufourt y Viallier (5), dicen que la difteria no ejerce ninguna acción sobre la tuberculosis.

Choffe (7). en su sanatorio de Hendaya, tevisa 286 difterias. la mayoría de ellas con antecedentes francos de tuberculosis, y en ninguno notó manifestaciones tuberculosas.

Aviragnet, Weil, Halle y P. L. Marie, dicen, en el tratado de Roger, Widal y Teissier (23), que la difteria suele ejercer a menudo acción desfavorable sobre la tuberculosis.

Nosotros hemos estudiado 22 casos de difteria, $y$ he aqui los resultados, sin mayores comentarios. 


\begin{tabular}{|c|c|c|c|}
\hline $\begin{array}{l}\text { N.P de } \\
\text { casos }\end{array}$ & & $\begin{array}{c}\text { Influídos } \\
\text { desfavorublemente }\end{array}$ & $\begin{array}{l}\text { No } \\
\text { inflaidos }\end{array}$ \\
\hline 18 & Con lesiones TBC anteriores.... & $3(14 \%)$ & $15(68 \%)$ \\
\hline 4 & $\begin{array}{l}\text { Aparición de lesiones TBC conse- } \\
\text { cutivamente a difteria }\end{array}$ & $4(18 \%)$ & \\
\hline 22 & TOTAL & $7(32 \%)$ & $15(68 \%)$ \\
\hline
\end{tabular}

Una vez más, debemos insistir sobre otros factores de agtavación: mala alimentación e higiene defectuosa especialmente. La mayoria de las agravaciones son sólo temporales; dos de ellos fallecieron. Como siempre, los casos agravados corresponden especialmente a las formas activas de tuberculosis, $v$ los no agravados a las formas inactivas.

\section{Parotiditis y taberculosis}

W. Schwenk (27), dice que la parotiditis epidémica ejerce una acción desfavorable sobre la tuberculosis, especial. mente sobre las formas infiltrativas, y en los niños que además tienen una constitución linfático-exudativa.

Dufourt y Viallier (5), creen que la parotiditis no ejerce ninguna acción sobre la tuberculosis.

Nalbant (9), estudiando 25 casos de parotiditis encontró algo inesperado: en los 25 hizo tuberculino-reacción antes y durante la parotiditis, y en 24 de ellos encontró una reacción más intensa durante la parotiditis que antes de ella; en un solo caso la reacción se hizo negativa. En ninguno de ellos se agravó la tuberculosis.

Choffe (7), en 354 casos de parotiditis epidémica observados en su sanatorio, no encuentra ninguna influencia de esta enfermedad sobre la tuberculosis activa o latente.

Nosotros estudiamos 8 casos de parotiditis epidémica, todos ellos aparecidos en el sanatorio para niños tubetculosos de "Los Guindos", del Consejo de Defensa del Niño; en ninguno de ellos observamos la más mínima influencia de la parotidit1s sobre la tuberculosis. Como vemos, nuestros resultados están de acuerdo esta vez con los que han obtenido la mayoría de los autores. 


\section{Tifus exantemático y tuberculosis}

Sobre esta materia no hemos encontrado absolutament? nada en la literatura mundial, lo que se explica fácilmente por el hecho de que el tifus exanternático es actualmente una çuriosidad en Europa y Estados Unidos.

Nosotros tenemos 4 casos, y no podemos sacar ninguna conclusión por ser muy reducido el número de observaciones. Sólo como dato ilustrativo diremos que, en un caso el tifus exantemático no influyó en el curso de la tuberculosis, y en los otros tres la tuberculosis apareció, aparentemente al menos. después del tifus exantemático; en los tres casos se trató de formas graves de tubetculosis y los tres evolucionaron hacia la muerte. Haremos notar, que a pesar de los numerosos casos de tifus exantemático que se presentaron en el año 1932 y siguientes, sólo en 4 casos encontramos, revisando la estadística respectiva, la asociación entre tuberculosis y tifus exante mático.

A continuación van dos casos clínicos.

CASO N. 18.-J. E. Obs. 1836. 1934, Edad: 1 año y dos meses.

Antecedentes.-No hay antecedentes de contagio tuberculo $\div 0$; ha sido sa. no basta ahora; criado en buenas condiciones dietéticas a bigiénicas.

Ingresa al Servicio de Infecciosos del Hospital Arriarán el 6 de julio de 1934. con el diagnóstico de tífus exantemático, que se compraeba on el sar. vicio taato clínica como serológicamente. Esta enfarmedad evolucionó en fortạa favorable. pero después de ella quedó si-mpre con fí:bre y tos, por lo que el 11 de agosto se toma la radiografia 5784, que revela la presencia die un completo primario del pulmón detecho. Esta lasión evolucionó desfayorablemente en forma moy rápida $y$ el enfermo fallece en poco más de dos meses. el in de srptiembre de 1934, con signos de condensación pu!monar. La baciloscopia fué negativa y el Pirquet positivo $\mathbf{4 0}$ dias después del tifus exantemático.

Autopsia: neumonia caseosa del tóbulo medio del pulmón derecho.

Comentario.-Lactante de 1 año y 2 meses de edad en el que, a raíz de un tifus exantemático se observa una neumonia caseosa de evolución fatal. En este caso. la influencia del tifus exantemático sobre la tuberculosis es cierta, a juzgar por el tipo de la lesión, que con seguridad habría dado síntomas generales y pulmonares si hubiera aparecido antes de contraer cl tifus exantemático. 
CASO N.9 19.-E. G. Obs. 2306. 1933. Edad: 4 años.

Antecedentes.- No se conoce la tuente de contagio tuberculoso; ha sita sana hatta ahora: criada en regulares condiciones higiénicas y dietéricas.

Ingresa al Servicio de Infecciosos del Hospita: Arriarán el 28 de septienbre de 1933 por tifus exantemático, que se comprueba con an Weil-Fé ix positiyo al 1/140; además preenta una otitis media supurada. derecha. Estuvo 16 días en el Servicio de Infecciosos, donde se encuent:an signos de condensación palmonar en el lóbalo superior izquierdo y un Pirquet poitivo. Por tales razones, traslada al Setvicio de Taberculosis para su ulterios observación. En esce servicio se diagnostica neumonia cazeosa, pero la radioscopia seña an probable pío-neumotórax enquistado que, a la punción da pas con bacilos de Korh al examen directo. Sin embargo, las últimas tadioscopias demuesran una destrucción extensa del tejido pnlmonar. La chica moere en caquexia el 6 de enem de 1934 .

Antopsia: caperna del lóbulo supetior izquicrdo, con lesiones caseosas d: Ins ganglios tráqueo-brónquicos $\mathrm{y}$ diseminación miliar genesalizada.

Comertatio.-Niña de 4 años, que contrae un tifus exantematico estando en un estado de salud aparentemente sa. tisfactorio; pero que a los pocos días de su estada en el hospital se le comprueban signos de condensación que llevan tápidamente a la enfermita a la caquexia y a la muerte. Es indudable que han existido lesiones tuberculosas antes de apa. recer el tifus exantemático, a juzgar por el carácter anátomopatológico de ellas, pero la enfermedad intercurrente ha producido una exacerbación rápida de esas lesiones con grandes destrucciones del parénquima y generalización miliar en otros organos.

\section{Erisipela y tuberculosis}

Tampoco tenemos ningún dato en la literatura sobre este lema, y sólo como dato ilustrativo diremos que en nues. tra estadística figuran dos casos de erisipela: en uno de ellos, la tuberculosis fué agravada por esta enfermedad y en el otro no se observaron modificaciones. No haremos ningún comentario sobre este tema, ya que de dos observaciones no podemos colegir nada.

A continuación analizaremos con detalle un caso especjal: se trata de una chica en la que actuaron tres enfermedades infecciosas: coqueluche, varicela y sarampión, y en la que es difícil precisar el papel jugado por cada una de ellas en la evolución de su tuberculosis. 
CASO N." 20.-.N. C. Obs, 1181. 1931. Edad: 3 años.

Antecedentes.-La madre falleció de tuberculosis pulmonar hide un ano. No se trenén más datos sobre ella.

Primera hospitalización.-Ingresa al Servicio de Tuberculosis cerrada del Mospital Arriarán, el 18 de junio de 1931, con un impétigo del caero cabeliudo y tha rinofaringitis. El examen pulmonar demuestra signos cacatrales difusos. Temperatura hasta 38\%. Pirquer positivo débil. Radioseopía totaxica negativa. Evolución subfibril. I.as lesion:s de impétigo y la rinofaringitis mejoxan considexabemente Pasa Iuego al Servicio de Coxueluche, por presentar ácesos tipicos de csa enfermedad. En csc scruicio sigue con signos catarrales primionares y temperatura snbfebril. La radiografia pulmonat N.9 636 describe un palmon coqueluchoso y ona plenritis costodiaftagmatica izquierda. Alta el 23 de ja'io de 1931. todavia con accesos de tos y un poco de fixbre por las tardes. fją pleuritis no dió signos clinicos.

Segunda bospitalización.-Ingtesa de nuevo al Servicio de Coquelache port sue ha seguido con acesos de tos $y$ ban aparecido numerosos elementos de vari(ela, el 11 de agosto de 1931. En el servicio sc comprueba una vaticela qu: sigue nua evolución penfigoidea y que por ultimo se impetiginiza, síndo muy rebelde al tratamiento. Las temperaturas sor sabfebrics; el axamen palmonar revela roncus, sibilancias $y$ algnnos estertores bronquiales. Una radioscopia $b_{x}$ cha el $1.9 \mathrm{~d}$ octubre demaestra an pulmón coqueluchoso $\mathrm{y}$ una pileurtis in rerlobar derecha. El Pirquet es intensamente positivo un mes y medio drspués de stu ingreso. Los accesos de tos se bacan muy intensos: disnea: temperatura vasta $40^{\circ}$. El examen pulmonat clinico y radiologico hecho ef 28 de octubre demuestra una pleuritis costodiafragmática bilateral y un proceso infittrativo det lóbrto medio inferior derecha. Esta pleuritis hace su evolnción siempre con temferatura ajta: el estado general desmejora: los aceesos dismituyen de frecuencis a intensidad: Pirquet intensamentz positivo en novismbre. El $17 \mathrm{de}$ no viembre de 1921 aparece un sarampión con abundantes signo; catartales putmonares. després del cual queda una temperatura hastante alta e intermirente: tos actesos coqueluchosos vuelven a aumentar en númeto e intensidad. Los sagnos pulmonares de p'euritis no varian. La radiografia N.' 1223 da igual imagen que los exámenes anteriones. Pasa al Servicio de Tuberculosis, donác tene una estada de 7 dias: un nuevo Pirquet es siempre positivo. Alea el 23 di diciembre de 1931, a pedido de la familia: sale stempre con temperatura alta muy disneica, decajida y con accesos de coqueluche.

Tercera hospitalización.-Ingresa al Servicio de Infecciosos el 19 de aneto de 1932, o sea, un mes despás de haber salido de Tuberculosis. con al euadro clinico de noa meningitis; el estadio del líquido céfalo-raquideo indica aqu se trata de una meningitis tubercalosa. El examen puluonat da una disminución del marmullo vesicular en la base izquierda, con broncofonia y soplo unspiratorio snave a ese nivel; franja de masidez en la base derecha. La radioscopía dice: grandes sombras, más o menos redondeaclas en la región hiliar de. recha; sombras irregulate en al hilio opuesto. Pirquet intansamente positiro. 
Hallece al 15 de febrero di 1932 .

Antopsia: tuberculosis miliar generaizada,

Comentario.-Niña de 3 años, observada en el Servicio de Tuberculosis por un Pirquet positivo, pero con exámenes radiológicos de tórax negativos. Contrae una coqueluche que evoluciona más o menos bien. Un mes después reingresa al hospital por una varicela de típo penfigoídeo. impetiginizada. E1 control radiológico en esta época, demuestra una infiltración del lóbulo medio derecho y pulmón coqueluchoso. Tres meses después, adquiere un sarampión que agrava considerablemente el cuadro clínico, aunque la imagen radiológica se mantiene más o menos igual. Poco después, se comprueba una meningitis tuberculosa, dínicamente y a la autopsia. Este caso tiene gran interés, porque se han podido observar en el niño tres enfermedades infecciosas sucesivas, con corto intervalo entre ellas. Respecto a la coqueluche, no podemos deducir ninguna influencia apreciable sobre la tuberculosis; en cambio, a raíz de la varicela, se pudo apreciar ya una infiltración del lóbulo medio derecho que, con el sarampión se disemina, dando origen a una miliar generalizada. Es difícil determinar con exactitud la participación que ha podido corresponderle a cada una de estas enfermedades; es posible que una haya preparado el terreno a la otra y es por eso que el sarampión aparece como el accidente final, determinante de la muerte. En todu caso, podemos apreciar muy bien en esta observación, la aparición y el desarrollo más o menos rápido de un proceso tuberculoso en un niño con diagnóstico de tuberculosis oculta, ocasionado por las tres enfermedades infecto-contagiosas que hemos descrito.

En el cuadro siguiente, exponemos gráficamente, $y$ en una forma fácil de consultar, los resultados generales de nuestra estadística: 


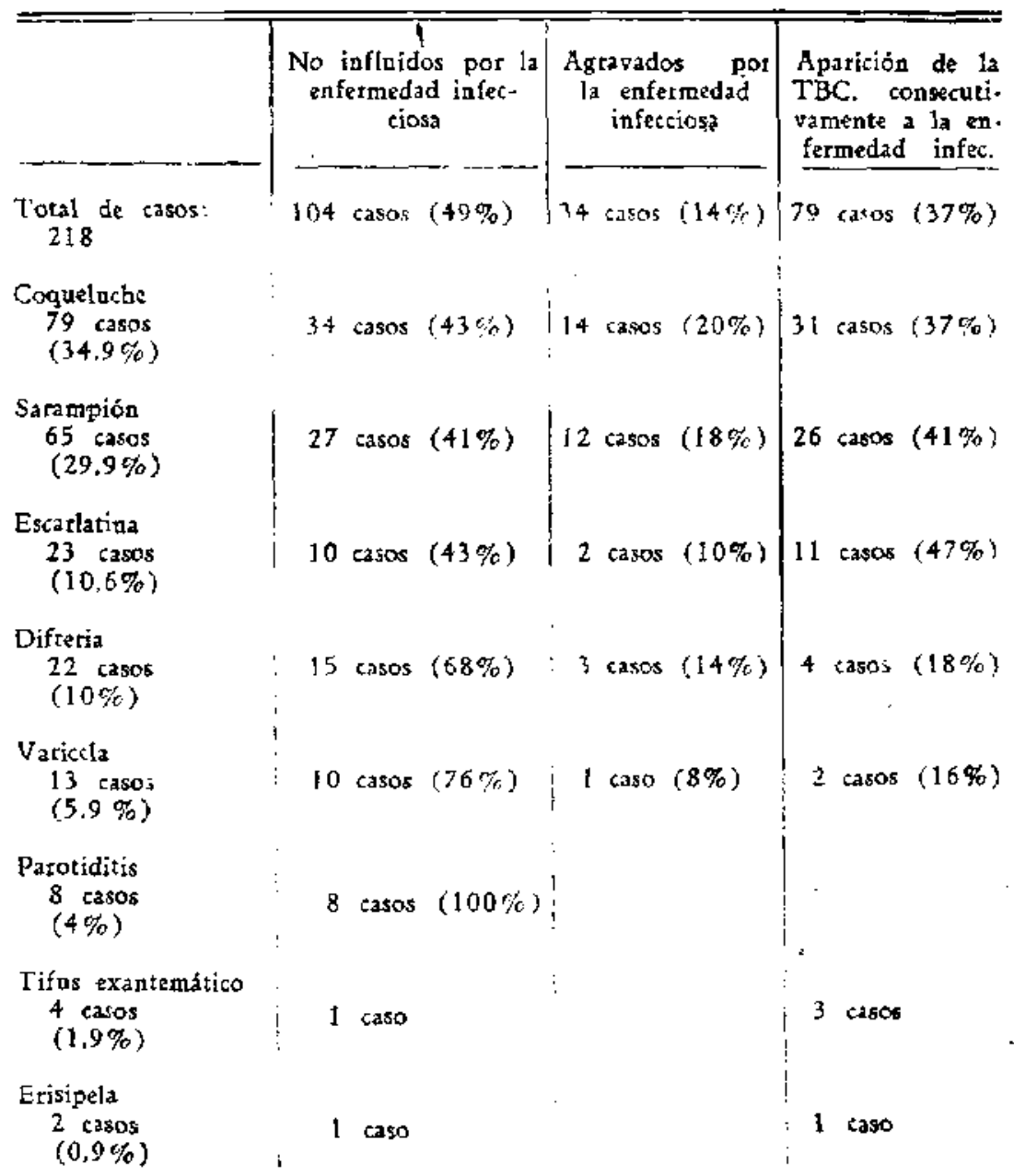




\section{Comentario general y conclusiones}

Podemos afirmar que los resultados de ǹuestras estadísticas, practicadas en 218 casos, nos demuestran que las enfermedades infecto-contagiosas tienen una influencia manifiesta sobre los procesos tuberculosos en el $50 \%$ de los casos aproximadamente, lo que nos coloca en un punto equidistante entre las dos tendencias extremas que existe sobre esta cuestion.

Analizando más detalladamente estos casos influídos por las enfermedades infecciosas, hemos podido comprobar que an $80 \%$ corresponde a formas francamente activas $\mathrm{y}$ progresivas, lo que demuestra que la agravación de la tuberculosis en estos enfermos está en relación directa con la actividad del proceso mismo. En el caso contrario, es decir, en aquellas tuberculosis no agravadas por la enfermedad intercurrente. encontramos una cifra sensiblemente igual de procesos inactivos $(85 \%)$.

Respecto a la localización de la lesión tuberculosa en nuestros enfermos, diremos que en el $70 \%$ era pulmonar, en el $20 \%$ mixta (pulmonar y ganglionar), y sólo en el $10 \%$ ganglionar pura.

Los enfermos agravados corresponden, en su mayor parte, a aquellos que han sido hospitalizados directamente en el Servicio de Infecciosos, sin exámenes previos pulmonares, 10 que estaría inđicando que estos niños no han seguido un tratamiento ni un régimen adecuado al contraer la enfermedad infecciosa, lo que se explica si tomamos en consideración que la tuberculosis infantil pasa a menudo inadvertida aun para los médicos, si no se dispone de antecedentes que orienten la investigación en este sentido. Debemos, pues, considerar estos casos como tuberculosos activos, en los cuales la enfermedad infecto-contagiosa ha determinado una agravación franca, a veces mortal.

Por otra parte, el grupo de los niños no agravados corresponde, en su mayor parte, a enfermos estudiados en el Servicio de Tuberculosis del Hospital Arriarán y en el Sanatorio de "Los Guindos"; donde se observó una epidemia de sarampión y otra de escariatina a mediados de 1938. Son las observaciones de este último establecimiento las que mejor demuestran la falta de influencia de las enfermedades infecciosas sobre la tuberculosis, que en muchos de ellos fué nula y en algunos sólo se observó una agravación de uno a dos meses de 
duración. Además, estos casos han sido observados clinica $Y$ radiológicamente, antes y después de la enfermedad infec. ciosa, $\sin$ que se apreciara en ellos una alteración aparente.

Otro factor que hemos analizado es el que se refiere a las condiciones hígiénico-dietéticas de las familias de estos niiios. Nuestra estadística al respecto nos indica que más del $50 \%$ de los niños provenian de hogares con malas condicio. nes higiénicas, y debemos recalcar el hecho de que precisamen10. fueron éstos los que tuvipron las mayores agravaciones.

$\mathrm{Si}$ analizamos las opiniones de los autores que hemos citado en los capitulos precedentes, nos encontramos con qui existe un verdadero antagonismo entre los clásicos y los modernos. Así, mientras que los primeros le atribuyen a las enfermedades infecciosas un papel funesto y a menudo mortal, cuando se asocian a la tuberculosis, los otros le dan muy poca importancia o no le dan ninguna, como los autores norteamericanos.

Nuestras investigaciones nos colocan en un término mecio entre estos dos grupos. La explicación de estos hechos s:ria, a nuestro juicio la siguiente: el diagnóstico de la tuber culosis, tanto del adulto como del niño, se hacía hasta hace algunos años, solamente por el examen clínico, y sabemos bien cuán escasa es la sintomatología clínica de la tuberculosis infantil en sus primeras etapas. El diagnóstico tenía que hacerse necesariamente tarde, cuando ya la lesión había llegado a un grado avanzado de desartollo y capaz de dar una sintomatología clínica evidente.

El estudio de la influencia que ejercen las enfermedades infecciosas sobre la tuberculosis se hacía de esta manera en enfermos en un grado avanzado de la enfermedad, en los cuales se producen, como ya hemos visto, las mayores agravaciones con las enfermedades infecto-contagiosas.

En el caso de los autores modernos, que ban trabajado con un material de diagnóstico más exacto (Rayos X. Labo. ratorios, etc.), estos estudios se han realizado en niños observados desde las primeras manifestaciones de la tuberculosis. que son las que menos se modifican con las enfermedades infecciosas.

En nuestros casos llegamos a una conclusión intermedia, porque nuestro material ha sido recogido en diversas fuentes: hospital, sanatorio, clientela privada. Ahora bien, si analizamos nuestras cifras separadamente, podemos comprobar que la casi totalidad de los casos agravados corresponde a enfermos con lesiones progresivas y a aquellos que se hospitalizaron por una enfermedad infecto-contagiosa, sin que se tu- 
viera noticias de la existencia de una tuberculosis anterior. Por otra parte, los enfermos del sanatorío "Los Guindos". donde aparecieron varias epidemias en 1938, (satampión, escarlatina, parotiditis, coqueluche), en ia sección convalecientes y los casos de la clientela privada, no experimentaron ninguna agravación con las enfermedades infecciosas.

Esto viene a demostrar que el factor enfermedad infecciosa no es decisivo en la agravación de la tuberculosis, sino que es preciso tomar en consideración más bien el tipo y la actividad de la lesión bacilar, junto con otros factores orgánicos o de ambiente que ya hemos analizado.

Resumiendo todo lo dicho hasta ahora, podemos exponer nuestras conclusiones en los siguientes puntos:

1) Sobre un total de 218 observaciones. se comprobó que en un $49 \%$ las enfermedades infecciosas no ejercian ninguna acción sobre la tuberculosis: en el $51 \%$ restante se pudo apreciar una influencia francamente desfavorable.

2) El sarampión, la coqueluche y la escarlatina agravan la tuberculosis en una proporción más o menos semejante: $59 \%$ para el sarampión. $57 \%$ para la coqueluche. $y$ $57 \%$ para la escarlatina. Estas agravaciones son a menudo temporales. La mortalidad para estas tres enfermedades alcanza a un $25 \%$.

3) La difteria ejerce una acción desfavorable sobre la tu. berculosis en el $32 \%$ de los casos, con una mortalidad del $10 \%$.

4) La varicela influye desfavorablemente a la tuberculosis en el $22 \%$ de los casos, pero en forma transitoria.

5) Respecto a las demás enfermedades infecciosas como parotiditis epidémica, tifus exantemático y erisipela, no podemos pronunciarnos. porque el número de casos is tudiados es insuficiente para ello.

6) Las enfermedades infecciosas agravan preferentemente las formas activas de la tuberculosis, especialmente las pulmonares.

7) Las malas condiciones higiénico-dietéticas de los niños que contraen una enfermedad infecto-contagiosa, tienen una gran importancia en la agravación de la tuberculosis.

8) Los casos de agravación de la tuberculosis se han obser vado principalmente en niños no examinados anteriormente desde este punto de vista, y que se hospitalizan por una enfermedad infecto-contagiosa. 


\section{BIBLIOGRAFIA}

1) V. de Lavergne.- "Allergie et anergie en clizique". G. Doin. Paris $193 \mathrm{i}$.

2) P. Nobicoutt, R. Liége et Mlle. A. Hen.-."Rougeole et tuberculose" Arch. de Méd. des enfants. Febreto 1930

9) P. Nobécourt.-"Maladies infecti.uses". Clinique Médicale aes enfants Mas:on. Paris 1933.

4) M. R. Goshrs.- "Apparition brusque d'adénites hi"aires tuberculeuses it la suite de rongeole". Bull. de la Soc. de Pédiatrie de Paris. Novinmbre 1936 .

5) A. Dufonrs ex Viallier.-"Au sujet de da prétendae infloence tuberculi. sante de la coquelache, de la rougeole et de la scatlation". Révae de ia tuberculose. Marzo 1937.

6) X. Dufourt et Viallier.-." $L$ s pouvoir tuberculisant de cirtaines maladiss infectienses". La Mèdecine. May̧o 1937.

7) Fréderic-Réné Choffe- "Du róle des maladies infectizuses dans l'étiologie de la tabetcu'o é". Thése de Paris. 1929.

8) J. D. Pilcher._-." Local skin ractions in measles and scarlet fevir in teJation to the intracutaneous taberculin reaction". The Am. Rev. of Tu. berculosis. Mayo 1935.

9) J. P. Nalbant.--"The effect of contagious diseases on pulmonary tubirculosis and on the tube:ca in reaction in children". The Am. Rev, of Tuberculosis. Diciembre 1937.

10) Herbert Koch.--"Matern und Tuberkulose". Arch. f. Kindern. Enaro 1933.

1.1) Frieda Bohning.- "Beobachtungen bei Masern". Arch. f. Kinderb. Febrero 1938.

12) Simon y Redeker.- "Manual práctico de tuberculosis infanti1". J. Mozata. Madtid 19?2.

13) Gabrieł.—"Pertussis und Tubetkulose". Jahrbuch f. Kinderh. Mayo 1934.

14) Alfred Sundal.-_Uebar Keuchusten, Beitrag za seiner Epidemiologie, Spmptomato'ogie and The:apie: Keuchuster und Tuberkaloie'. Act. Paediatrica, 30 noviembre 1932.

15) Roger Amsler et Engéne Ruais.-_Les rapports de la coque'uche et de da tuberculose chez l'enfant". Monde Médical. Enero 1936.

16) Léon Bernatd, Matrice Lamy et Mlle. Dumans.- 'La prétendue influence de la coqueluche sur la taberculose du nourrisson". Presse Médicale. 21 de septiembre 1932.

17) Ivan Hershey and Frieda Ward.-Whooping-Gough in roberculosis children". The Am. Rav. of Tuberculozis. Noviembre 1935.

18) C. Diez Fernández.- "La tuberculosis infantil coqueluchoide". La Medicina Ibériça, 8 de noviembre 1931. 
19) Olivio Ahumada.-"Dos años al estudio de la coquelacbe". Archivors del "Hospital de Niñ'os Manuel Arriatán". 1935.

20) L. Piccaluga.- "Rapporti fra scarlatinna e tnberculoci". Riv. di Clinica Pediatrica. Fasc. X. O. H. 1933.

21) Millian et Vassiliu.- "Tubetsulose et scarlatine". Bull. de la Soc, de Péliatrie de Paris. 1930. pág. 461.

22) O. Millian.- "La' tuberculose, facteur de malignité danj la scarlatine". Arch. de Méd, des eńfants. Marzo 1932.

23) Roger, Widal, Teissier.— "Nouveau Traité de Médecine". 2," edición. Vol. II, pág. 749. Masson Paris.

24) Georg Abraham.-- "Varicela y tuberca'osis". Monatsch. f. Kinderh. Fobre:o 1930 .

25) W. Scbwenk.--' $\mathrm{La}$ influencia de la varicela en el curso de la tuberculosis, en los niños". Ztich. f. Kinderh. 49:686. 19?0.

26) Generoso H. Schiavone- - Meningitis tubercalosa concecutiva a una va. ricela: ¿es la va:icela una enfermedad anergizantep". La Semana Médica. 21 de novizmbre 1929 ,

17) W. Schwenk.-"Kam Die kindliche Tuberku'o:e durch eine Parotitis epidemica beeinflust warden?". Archiv. f. Kindetb. Vol. 92, 1. I. 1930.

Jbras consultadas, pero no ciradas:

?. Nobécontt, L. Babonneix.-“"Traité de Médecine de; enfants". Masson it Cie. Paris 1934.

'faundler y Schlosamann.- "Tratado enciclopédico de enfermedades de la infancia". 4" edición. Fro. Seix. Barcelona 1932. 